\title{
Cyclophosphamide for Rapid-Onset Obesity, Hypothalamic Dysfunction, Hypoventilation, and Autonomic Dysregulation Syndrome
}

\author{
Ido Paz-Priel, MD, David W. Cooke, MD, and Allen R. Chen, MD, PhD \\ Division of Pediatric Oncology (I.P.-P., A.C.) and Division of Pediatric Endocrinology (D.C.), \\ Johns Hopkins University, Baltimore, MD
}

\section{Abstract \\ Patients with rapid-onset obesity, hypothalamic dysfunction, hypoventilation, autonomic dysregulation, and neural crest tumor syndrome have poor long-term outcomes. We report a patient who was treated successfully with high-dose cyclophosphamide immunoablation. This experience offers a novel therapeutic approach and an indirect insight into the underlying pathogenesis of this syndrome.}

\begin{abstract}
Asyndrome characterized by rapid onset of obesity, hypothalamic dysfunction, hypoventilation, autonomic dysregulation (ROHHAD), and neural crest tumors has been recently identified. ${ }^{1}$ Although the spectrum of this syndrome's manifestations has been described, ${ }^{1-3}$ the underlying pathogenesis is not clear, and an extensive effort to sequence candidate genes, including $P H O X 2 B$, was unrevealing. ${ }^{2,3}$ The association with neural crest tumors suggests a paraneoplastic, autoimmune etiology; this has not yet been confirmed, however. ${ }^{4}$ Many patients with ROHHAD succumb to respiratory failure or sudden death, and survivors experience debilitating sociocognitive deficits. ${ }^{1-7}$ Better therapeutic approaches are needed.
\end{abstract}

Because of its pharmacologic characteristics, high-dose cyclophosphamide results in neartotal ablation of lymphocytes and profound immunosuppression, with sparing of hematopoietic stem cells due to abundant expression of aldehyde dehydrogenase. ${ }^{8,9}$ Highdose cyclophosphamide without hematopoietic stem cell support was pioneered as treatment for severe aplastic anemia. Recent reports have demonstrated its efficacy in severe refractory autoimmune diseases, including autoimmune hemolytic anemia, systemic lupus erythematosus, multiple sclerosis, myasthenia gravis, and other autoimmune neurologic diseases. ${ }^{8-10}$ Importantly, high-dose cyclophosphamide is well tolerated; patients experience transient myelospression and recover from neutropenia by day 6-22. Thus, autologous hematopoietic stem cell support is not necessary, and avoiding it eliminates the risk of reinfusing autoreactive immune effector cells. This experience was recently reviewed in detail. ${ }^{8-10}$

We report a child with ROHHAD syndrome who had a reproducible, transient response to conventional immunosupression combined with rituximab and a sustained response to highdose cyclophosphamide.

Copyright () 2010 Mosby Inc. All rights reserved.

Reprint requests: Ido Paz-Priel, MD, Bunting-Blaustein Cancer Research Building I, Room 208, 1650 Orleans Street, Baltimore, MD 21231. ipazpri1@jhmi.edu..

I.P.-P. is a St. Baldrick's Foundation Scholar. The authors declare no conflicts of interest. 


\section{Case Description}

A 3-year, 10-month-old girl presented with hyperphagia and rapid weight gain. After growth at the 50th percentile up to age 2 years, her body mass index increased from $16.8 \mathrm{~kg} / \mathrm{m}^{2}$ (standard deviation [SD], 0.0) to $20.7 \mathrm{~kg} / \mathrm{m}^{2}$ (SD, 2.45) at age 30 months, and then to 25.4 $\mathrm{kg} / \mathrm{m}^{2}(\mathrm{SD}, 6.18)$ at age 36 months. This was associated with aggressive food-seeking behavior, abnormal sleep patterns, hypertension, decreased pain sensitivity, irritability, aggressive behavior, loss of toilet training, diffuse sweating without temperature instability, and left eye exotropia. Her serum prolactin level was $76.5 \mathrm{ng} / \mathrm{mL}$, and her thyroid and adrenal gland function were normal. Computed tomography revealed a retroperitoneal tumor, which was resected and identified as a ganglioneuroblastoma with favorable biological features. No further therapy for this neoplasm was indicated.

Brain magnetic resonance imaging and cerebrospinal fluid analysis were normal, and no oligoclonal immunoglobulin $\mathrm{G}$ was detected. A sleep study did not demonstrate hypoventilation.

This presentation is compatible with ROHHAD syndrome, although genetic testing for alternative diagnoses is unavailable. Because of the associated ganglioneuroblastoma, an immune-mediated process, akin to opsoclonus myoclonus ataxia (OMA) syndrome, was presumed. The patient was treated with cyclophosphamide, $750 \mathrm{mg} / \mathrm{m}^{2} /$ dose every 28 days for 6 doses; intravenous immunoglobulin, $1 \mathrm{~g} / \mathrm{kg} /$ dose every 28 days for 8 doses and then every 56 days for 2 additional doses; and prednisone, $2 \mathrm{mg} / \mathrm{kg} /$ day for 28 days, followed by a 20 -week-long taper. Five weekly doses of rituximab, $375 \mathrm{mg} / \mathrm{m}^{2} /$ dose, were added based on previous reports of failure of conventional dose immunosuppression ${ }^{4,5}$ and the utility of this agent in OMA. ${ }^{11}$

Within 2-3 weeks from the initiation of therapy, the patient improved, as evidenced by diminishment of appetite and stabilization of weight, normalization of sleep pattern, regaining of toilet training, reacquisition of premorbid personality, and resolution of the diffuse sweating episodes. After 3 months of improvement, while receiving intravenous immunoglobulin, cyclophosphamide, and prednisone taper, the patient's symptoms gradually recurred, and she returned to her pretreatment condition. A 3-month-long therapeutic trial of cyclosporine A produced no response.

Because of the patient's initial response to rituximab, a second course of five weekly doses was started. As before, a significant improvement was noticed after the second dose; however, the symptoms recurred within 3 weeks. Importantly, throughout the patient's course of illness, including before her transfer to our institution, she experienced continuous worsening of the ROHHAD manifestations without spontaneous improvement.

Because of the patient's reproducible response to immunosupression and her continuous deterioration, informed consent was obtained, and treatment was provided with cyclophosphamide $50 \mathrm{mg} / \mathrm{kg}$ ideal weight/day on 4 consecutive days. This therapy was well tolerated, with only a brief episode of uncomplicated neutropenia that resolved by day 10 . Starting 3 weeks after treatment, the patient demonstrated progressive improvement, with gradual reestablishment of toilet training; regaining of a pleasant, playful personality with appropriate emotional responses; improved sleep patterns; and resolution of aggressive food-seeking behavior, although she appears to lack a normal satiety response. At 10 months after treatment, the patient's weight had remained stable despite a 4-cm height increase. She was attended school for the first time and at her peers' level. 


\section{Discussion}

We report a patient with ROHHAD who had a favorable response to immunoablative treatment with high-dose cyclophosphamide. Our patient failed conventional-dose immunosupression, as previously reported, ${ }^{4,5}$ and experienced only short-lived relief from rituximab. High-dose cyclophosphamide "reboots" the immune system by ablating the mature immune elements and has been found to be efficacious in patients who fail conventional immunosuppression. ${ }^{8,9}$ The acute toxicity of this regimen is well tolerated, ${ }^{8,9}$ and quality-of-life measures compare favorably with those associated with conventionaldose cyclophosphamide. ${ }^{12}$ However, potential risks, including infection, infertility, and secondary neoplasm, must be considered. Our patient markedly improved after high-dose cyclophosphamide, albeit with limited follow-up. Given the progressive deterioration of most patients with ROHHAD, our experience is encouraging, and in carefully selected patients, this therapy may balance the potential risks of intensive immunosuppression.

The etiology of ROHHAD is not clear but has been speculated to be an autoimmunemediated process distinct from congenital central hypoventilation syndrome. ${ }^{1,2,4,5}$ On two separate occasions, our patient experienced symptomatic improvement shortly after initiation of rituximab therapy, but her symptoms recurred with waning of the immunosuppressive effect. A longer remission was achieved after high-dose cyclophosphamide induced immunoablation. The pattern that we describe fulfills the temporality, consistency, and dose-response criteria of causality. ${ }^{13}$ Moreover, an autoimmune process is biologically plausible, given that neuroblastoma is associated with autoimmune-mediated paraneoplastic syndromes, such as OMA syndrome. OMA syndrome and ROHHAD syndrome persist after complete tumor resection, pointing to an autoimmune process that is initiated by neuroblastoma but maintained in its absence. In addition, autopsies of patients with ROHHAD have revealed lymphocytic infiltration of the brain. 6,7 Thus, our patient's course indicates, albeit indirectly, an autoimmune mechanism in ROHHAD. However, ROHHAD syndrome may include etiologically diverse subgroups of patients, and autoimmunity may not be universally relevant.

Although spontaneous improvement cannot be excluded, it is less likely in our patient, who experienced continuous deterioration except for the 3 occasions when initiation of immunosuppression was associated with rapid amelioration of ROHHAD manifestations.

There is no gold standard for diagnosing ROHHAD, and our patient displayed many of its features with the exception of hypoventilation. This does not exclude ROHHAD, however, because hypoventilation is a late symptom, and its incidence might be overestimated by its inclusion in the case definition of previous reports and by an obvious diagnostic bias of patients with severe respiratory compromise. ${ }^{1,2}$ Thus, our observations may be applicable to other patients with ROHHAD as well. Ideally, early, effective intervention can halt the progression of this devastating complication.

Our patient provides an indication that, at least in a subgroup of patients, ROHHAD syndrome has a significant autoimmune component, and that intensive immunosuppression might be beneficial. These preliminary observations should be generalized with caution, and because of its potential toxicity, high-dose cyclophosphamide should be considered only in carefully selected patients. Longer follow-up is needed, and our experience should be reproduced, ideally in a collaborative study.

\section{Glossary}

OMA Opsoclonus myoclonus ataxia 
ROHHAD Rapid onset of obesity, hypothalamic dysfunction, hypoventilation, autonomic dysregulation

SD

Standard deviation

\section{References}

1. Bougneres P, Pantalone L, Linglart A, Rothenbuhler A, Le Stunff C. Endocrine manifestations of the rapid-onset obesity with hypoventilation, hypothalamic, autonomic dysregulation, and neural tumor syndrome in childhood. J Clin Endocrinol Metab. 2008; 93:3971-80. [PubMed: 18628522]

2. De Pontual L, Trochet D, Caillat-Zucman S, Abou Shenab OA, Bougneres P, Crow Y, et al. Delineation of late-onset hypoventilation associated with hypothalamic dysfunction syndrome. Pediatr Res. 2008; 64:689-94. [PubMed: 18670370]

3. Ize-Ludlow D, Gray JA, Sperling MA, Berry-Kravis EM, Milunsky JM, Farooqi IS, et al. Rapidonset obesity with hypothalamic dysfunction, hypoventilation, and autonomic dysregulation presenting in childhood. Pediatrics. 2007; 120:e179-88. [PubMed: 17606542]

4. Sirvent N, Berard E, Chastagner P, Feillet F, Wagner K, Sommelet D. Hypothalamic dysfunction associated with neuroblastoma: evidence for a new paraneoplastic syndrome? Med Pediatr Oncol. 2003; 40:326-8. [PubMed: 12652624]

5. Katz ES, McGrath S, Marcus CL. Late-onset central hypoventilation with hypothalamic dysfunction: a distinct clinical syndrome. Pediatr Pulmonol. 2000; 29:62-8. [PubMed: 10613788]

6. North KN, Ouvrier RA, McLean CA, Hopkins IJ. Idiopathic hypothalamic dysfunction with dilated unresponsive pupils: report of two cases. J Child Neurol. 1994; 9:320-5. [PubMed: 7930414]

7. Ouvrier R, Nunn K, Sprague T, McLean C, Arbuckle S, Hopkins I, et al. Idiopathic hypothalamic dysfunction: a paraneoplastic syndrome? Lancet. 1995; 346:1298. [PubMed: 7475740]

8. Brodsky RA. High-dose cyclophosphamide for autoimmunity and alloimmunity. Immunol Res. 2010; 47:179-84. [PubMed: 20087683]

9. Brodsky RA, Jones RJ. Intensive immunosuppression with high-dose cyclophosphamide but without stem cell rescue for severe autoimmunity: advantages and disadvantages. Autoimmunity. 2008; 41:596-600. [PubMed: 18958751]

10. Drachman DB, Brodsky RA. High-dose therapy for autoimmune neurologic diseases. Curr Opin Oncol. 2005; 17:83-8. [PubMed: 15725909]

11. Pranzatelli MR, Tate ED, Travelstead AL, Barbosa J, Bergamini RA, Civitello L, et al. Rituximab (anti-CD20) adjunctive therapy for opsoclonus-myoclonus syndrome. J Pediatr Hematol Oncol. 2006; 28:585-93. [PubMed: 17006265]

12. Dussan KB, Magder L, Brodsky RA, Jones RJ, Petri M. High-dose cyclophosphamide performs better than monthly-dose cyclophosphamide in quality of life measures. Lupus. 2008; 17:1079-85. [PubMed: 19029275]

13. Weed DL. On the use of causal criteria. Int J Epidemiol. 1997; 26:1137-41. [PubMed: 9447391] 